\title{
SPECIMEN OF UNREDUCED DISLOCATION BACKWARDS OF THE BONES OF THE FOREARM.
}

\author{
By EDWARD H. BENNETT, M.D., Dubl. ; F.R.C.S.; \\ Professor of Surgery in the University of Dublin ; \\ Surgeon to Sir Patrick Dun's Hospital.
}

[Read in the Section of Pathology, May 4, 1888.]

I BEG leave to exhibit a specimen of unreduced dislocation of the bones of the forearm backwards, preserved in spirit, and with it six similar specimens, preserved after maceration. My object in bringing forward these specimens is to draw attention to the fact that, although the varieties and conditions of elbow-joint dislocation have been very fully described, yet still, as regards the point with which $I$ am about to deal, the descriptions of this commonest of dislocations are, even at the present day, such as are calculated to mislead. They tend to errors of diagnosis. Take Erichsen's description of this lesion :- "The most common dislocation is that in which both bones are thrown backwards, with or without fracture of the coronoid process. This injury is readily recognised by the projection backwards of the olecranon, carrying with it the tendon of the triceps. The articular end of the humerus also can be felt projecting in front of the elbow. When the coronoid process is not broken off it is fixed against the posterior surface of the humerus, the forearm being immovably placed in its new position. When this process is fractured there is great mobility about the joint, and crepitation may be felt as the arm is drawn forwards." I now place before the Academy three specimens in which there is complete dislocation of both bones backwards, the coronoid process resting behind the trochlea of the humerus. 'To these such a description as that $I$ have quoted from Erichsen 
applies exactly, but it necessarily excludes every other variety of dislocation of both bones backwards. It ignores such a thing as incomplete dislocation backwards of the bones of the forearm, of which four examples are exhibited. Incomplete dislocation was first described, with great care-as far as exact description could go-by Malgaigne, but it has been long a subject of discussion as to whether it was known to Hippocrates. The important feature of the incomplete, as contrasted with the complete, is briefly given by Littré :- "The incomplete luxation backwards is the only one in which the olecranon does not appear sensibly altered in position." Denucé, following Malgaigne, has stated clearly the pathological difference between incomplete and complete dislocation, putting it in a very few words, and pointing out in incomplete dislocation the presence of the coronoid process at the level of the trochlea. In the specimen of incomplete dislocation preserved with the soft tissues dissected, as well as in the three other old specimens now before the Academy, the hardly appreciable displacement of the olecranon is well marked. The following description of the injury given by Hamilton, the chief of American authorities, is:- "The relation of the olecranon process also to the condyle is changed, and the upper end of this process, instead of being a little below the internal condyle, as it would be naturally when the arm is slightly flexed, is found carried upwards towards the shoulder, from half an inch or more, above the condyle." From the conditions seen in any one of the specimens, it is clear that in incomplete dislocation no such change in the position of the olecranon occurs. Hamilton makes no allusion whatever to the incomplete dislocation. Another authority of great weightSouth-said: "The dislocation backwards is the most common and always the most complete. The coronoid process of the cubit gets behind the pulley-like joint surface of the upper arm bone and rests in the pit formed by the olecranon." That was a description of complete dislocation, no mention being made of incomplete. One of the most recent writers-Treves-in his recently-issued book, says :- "Dislocations of the elbow of all kinds may be partial or complete. More usually they are complete when in the antero- 


\section{Unreduced Dislocation Backwards of the Forearm.}

posterior direction, and partial when the luxation is lateral." That almost excludes the existence of the incomplete dislocation backwards. It was admitted by Malgaigne, and those who studied the lesion about the time that he wrote, that incomplete dislocation of the elbow was more common than complete dislocation. Inconıplete dislocations, such as are illustrated by the recent and dry specimens before the Academy, have been allowed to go unreduced because they have not been recognised. Such error arises in great measure from reliance on the test given by the authorities quoted-the displacement of the olecranon above the line connecting the condyles of the humerus. This displacement is present in complete dislocations, but it is absent in the incomplete. To ignore the existence of the incomplete dislocations, or to teach that they are recognised by displacement of the olecranon, " half an inch or more above the condyle," is to insure that they will remain unreduced. Now, it is admitted that backward dislocations of the elbow are the most common variety of displacement of the joint, and of these the incomplete are the more common. In museums, at all events, the latter are the more common, perhaps because they are those most frequently left unreduced. In the moist preparation before the Academy no doubt can exist as to the relative position of the bones-a doubt that some might entertain in the case of the macerated specimens. In the words of Malgaigne, " the projection of the olecranon is on a plane sensibly beneath the projection of the epitrochlea, whilst in the complete luxation the olecranon is placed sensibly ahove that process." My attention having been directed to the subject by obtaining this recent specimen, I have thought it well to reiterate the statement of Malgaignethough it was sufficiently distinct, and though it ought to be familiar to writers on the subject - that the incomplete form of dislocation backwards at the elbow does occur; that it is the more common form of dislocation of both bones, which, by being left unreduced, subsequently become museum specimens; and that it probably has in these remained unrecognised because the test of the elevation of the olecranon, relied on in the descriptions of complete dislocation, is applied to it. It is very easy for an in- 
complete dislocation to be overlooked when a certain amount of swclling conceals the rarious features, including the existence of the tumour formed by the displaced humerus in front, and the abnormal projection of the head of the radius backwards. The specimen before the Academy exhibits these peculiar characteristics of partial dislocation; in fact, looking at the back of the elbow, one would say, at first sight, that there was no dislocation at all of the ulna. But the radial dislocation catches the eye, and in front the humerus projects as an unmistakable tumour. 